\title{
Optimization of directional elastic energy propagation
}

\author{
Andreassen, Erik; Chang, Hannah R.; Ruzzene, Massimo; Jensen, Jakob Søndergaard
}

Published in:

Journal of Sound and Vibration

Link to article, DOI:

10.1016/j.jsv.2016.03.002

Publication date:

2016

Document Version

Peer reviewed version

Link back to DTU Orbit

Citation (APA):

Andreassen, E., Chang, H. R., Ruzzene, M., \& Jensen, J. S. (2016). Optimization of directional elastic energy propagation. Journal of Sound and Vibration, 379, 53-70. https://doi.org/10.1016/j.jsv.2016.03.002

\section{General rights}

Copyright and moral rights for the publications made accessible in the public portal are retained by the authors and/or other copyright owners and it is a condition of accessing publications that users recognise and abide by the legal requirements associated with these rights.

- Users may download and print one copy of any publication from the public portal for the purpose of private study or research.

- You may not further distribute the material or use it for any profit-making activity or commercial gain

- You may freely distribute the URL identifying the publication in the public portal

If you believe that this document breaches copyright please contact us providing details, and we will remove access to the work immediately and investigate your claim. 


\title{
Optimization of directional elastic energy propagation
}

\author{
Erik Andreassen $^{\mathrm{a}, *}$, Hannah R. Chang ${ }^{\mathrm{b}}$, Massimo Ruzzene ${ }^{\mathrm{b}}$, Jakob Søndergaard Jensen $^{\mathrm{a}}$ \\ ${ }^{a}$ Department of Mechanical Engineering, Technical University of Denmark, Nils Koppels Allé, Building 404, Denmark \\ ${ }^{b}$ School of Aerospace Engineering, Georgia Institute of Technology, Atlanta, GA 30332
}

\begin{abstract}
The aim of this paper is to demonstrate how topology optimization can be used to design a periodically perforated plate, in order to obtain a tailored anisotropic group velocity profile. The main method is demonstrated on both low and high frequency bending wave propagation in an aluminum plate, but is general in the sense that it could be used to design periodic structures with frequency dependent group velocity profiles for any kind of elastic wave propagation. With the proposed method the resulting design is manufacturable. Measurements on an optimized design compare excellently with the numerical results.
\end{abstract}

Keywords: wave propagation, perforated plate, microstructure, topology optimization, phononic crystal, group velocity

\section{Introduction}

Wave propagation in an elastic medium depends on the stiffness and density distribution. The governing equation for linear elastic wave propagation is:

$$
\rho \frac{\partial^{2} \mathbf{u}}{\partial t^{2}}=\partial^{T} \mathbf{C} \partial \mathbf{u}
$$

where $\rho$ is the density, $\mathbf{u}$ is the displacement field, $t$ is time, $\boldsymbol{\partial}$ is the spatial derivative operator, and $\mathbf{C}$ is the constitutive matrix.

By periodic variations in the density or stiffness $(\mathbf{C})$ of the structure, one can achieve unique wave propagation properties, such as bandgaps [1], wave beaming [2], and negative refraction $[3,4,5]$. In photonics, where analogous phenomena can be achieved by periodic variations in optical properties, a phenomenon called slow light [6], where the group velocity of a light pulse is lower than the speed of light, has received considerable attention. While Cox and Dobson [7] were some of the first to use topology optimization to design photonic bandgap structures, in [8] it is illustrated how topology optimization [9] can be used to design a photonic crystal with tailored group velocity properties.

Returning to elastic wave propagation; for materials where dissipation is negligible, the group velocity corresponds to the energy velocity [10], and controlling how the energy propagates have several possible applications. A simple and obvious one is vibration shielding, but it could also be used in more advanced applications, such as energy harvesting [11].

For a periodic structure the wave solution to the full dynamic problem in Eq. 1 can be expanded according to the Bloch theorem [10]:

$$
\mathbf{u}(\mathbf{x}, t)=\tilde{\mathbf{u}}(\mathbf{x}) e^{i \mathbf{k}^{T} \mathbf{x}} e^{i \omega t}
$$

where $\tilde{\mathbf{u}}$ is the periodic displacement field, $\mathbf{k}=\left(k_{x}, k_{y}, k_{z}\right)^{T}$ is the wave vector, and $\omega$ is the temporal frequency. This allows

*E-mail: erand@mek.dtu.dk a characterization of the dynamic properties by analysing the unit cell. Inserting Eq. (2) into Eq. (1) will yield an eigenvalue problem on the unit cell, which, as shown by the authors in an earlier paper [12], can be used to compute the group velocity $\mathbf{c}_{g}$ for a given frequency $\omega$.

The aim of this paper is to present a method, based on topology optimization, for tailoring the group velocity profile in a structure by creating a periodic pattern in the structure. The method will be demonstrated with the design of a perforated plate. The goal is to make flexural (transverse) waves propagate faster in one direction.

Focusing on a plate structure, as opposed to a full threedimensional structure, keeps the computational burden relatively low. Furthermore, it makes an experimental verification simpler, because the flexural wave propagation in a perforated plate can easily be measured using an optical measuring technique, such as a scanning laser vibrometer (SLV). That said, the presented method is also applicable to three-dimensional problems.

As already mentioned, the gradient-based method called topology optimization will be used to tailor the unit cell design. In this paper we present methods to tailor the group velocity profile, while the focus of most of the earlier work in elastic wave propagation in periodic structures has been maximization of bandgaps. The first to apply topology optimization to design periodic structures with elastic wave propagation in mind were Sigmund and Jensen [13]. Halkjær et al. [14, 15] used topology optimization to design unit cells for maximum bending wave bandgaps. Similarly, El-Sabbagh et al. [16] maximized the bandgaps for bending wave propagation, but considered a finite plate with a finite periodic pattern. While Larsen et al. [17] optimized the wave propagation pattern in a finite plate, without requiring a periodic pattern in the plate.

We start by presenting the optimization problem in Section 2 , where the numerical modelling is also discussed. In Section 3 several optimized designs are presented and discussed. Furthermore, one of the designs have been manufactured by per- 


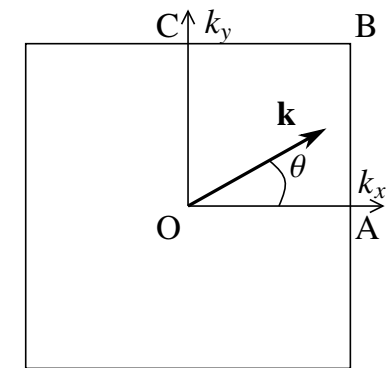

Figure 1: Illustration of the Brillouin zone and wave vector direction specified by the angle $\theta$.

forating an aluminum plate, and the experimental results from SLV measurements are compared to the numerical predictions in Section 4.

\section{Theory}

\subsection{Group velocity of elastic waves}

The derivation of the equations necessary to compute the group velocity profile (for flexural waves in a plate with a periodic pattern) is described in detail in [12], and it will not be repeated here. Numerically, the group velocity profile at a given frequency $\omega$ can be computed by first finding the wave vectors by solving the discretized eigenvalue problem

$$
\left(\mathbf{K}_{k}(\theta, \omega)-k \mathbf{M}_{k}(\theta)\right) \mathbf{u}_{k}=\mathbf{0},
$$

where $\theta$ is the angle between the wave vector components $k_{x}$ and $k_{y}, \omega$ is the temporal frequency and $k=|\mathbf{k}|$ is the wave vector length, and the finite element matrices $\mathbf{K}_{k}$ and $\mathbf{M}_{k}$ for a Mindlin plate problem are given in Appendix A. Figure 1 provides a basic illustration of the wave vector and its components along with an indication of the irreducible Brillouin zone corresponding to the repetitive unit cell (see e.g. [10] for a fundamental explanation of Brillouin zones for periodic media).

For a given combination of $\theta$ and $k$ the group velocity $\mathbf{c}_{g}=$ $\left(c_{g x}, c_{g y}\right)^{T}$ is given as

$$
\begin{aligned}
& c_{g x}=\frac{1}{2 \omega} \frac{\mathbf{v}^{T}\left(-i \mathbf{K}_{1}+2 k \cos (\theta) \mathbf{K}_{3}+k \sin (\theta) \mathbf{K}_{4}\right) \mathbf{u}}{\mathbf{v}^{T} \mathbf{M u}}, \\
& c_{g y}=\frac{1}{2 \omega} \frac{\mathbf{v}^{T}\left(-i \mathbf{K}_{2}+2 k \sin (\theta) \mathbf{K}_{5}+k \cos (\theta) \mathbf{K}_{4}\right) \mathbf{u}}{\mathbf{v}^{T} \mathbf{M u}},
\end{aligned}
$$

where the computations of the matrices $\mathbf{K}_{i}$ and $\mathbf{M}$ are detailed in Appendix A. $\mathbf{u}$ can be found from $\mathbf{u}_{k}$ (the solution to Eq. 3), which is defined as

$$
\mathbf{u}_{k}=\left[\begin{array}{c}
k \mathbf{u} \\
\mathbf{u}
\end{array}\right],
$$

while $\mathbf{v}$ is $\mathbf{u}$ complex conjugated counterpart. If dissipation were to be included, obtaining $\mathbf{v}$ would require the solution of an additional eigenvalue problem.
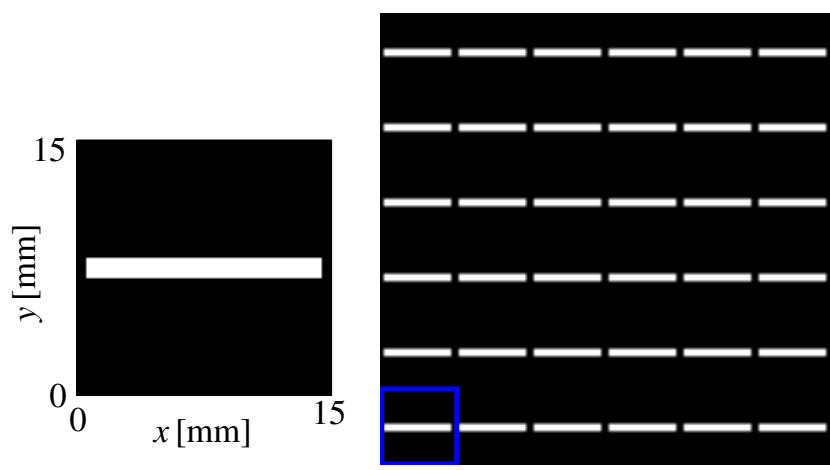

Figure 2: An intuitive design for a unit cell of a plate structure with a high $f_{0}$ for low frequencies. Left: One unit cell. Right: An assembly of six times six unit cells.

\subsection{Performance measure}

It is important to remark that $\mathbf{c}_{g}$ does not necessarily point in the same direction as k. However, when $\theta=0^{\circ}$ and $\theta=90^{\circ}$ the two point in the same direction. Utilizing this makes it simpler to define the performance measure for the optimized designs. In order to get a clear directional dependency, we can maximize the ratio between the group speed ${ }^{1}$ in these two perpendicular directions. Thus, we define the performance measure as

$$
f_{0}=\operatorname{sign}\left(\frac{\left.c_{g x}\right|_{\theta=0}}{\left.c_{g y}\right|_{\theta=\pi / 2}}\right) \cdot \frac{\left.c_{g x}\right|_{\theta=0}}{\left.c_{g y}\right|_{\theta=\pi / 2}} .
$$

This ratio will vary with the considered frequency, but for a range of low frequencies (wave lengths larger than the periodic features) this ratio is well-defined and will vary slowly. We will return to the problem of higher frequencies shortly.

\subsection{Benchmark design}

In order to evaluate the performance of the optimized designs, we introduce an intuitive design, which will serve as a benchmark. We take outset in a unit cell of 15 times $15 \mathrm{~mm}$ and with a thickness of $2 \mathrm{~mm}$. The final plate structure will be manufactured in aluminum (Young's modulus $E=69 \mathrm{GPa}$, Poisson's ratio $v=0.33$, and the density $\rho=2700 \mathrm{~kg} / \mathrm{m}^{3}$ ) using water jetting, with a minimum required width of holes and structural members of $1.2 \mathrm{~mm}$ (for the specified thickness). To maximize $f_{0}$ for low frequency waves, we assume one can try to block the waves from propagating in the $y$-direction by making a long thin cut in the $x$-direction. This is illustrated in Fig. 2 , where a unit cell consists of an air gap with the minimum width of $1.2 \mathrm{~mm}$ in the vertical direction is illustrated. Thin rods, also with the minimum width of $1.2 \mathrm{~mm}$, hold the design together in the vertical direction. Remark, throughout the paper black regions symbolizes solid material (aluminum), while white regions symbolizes void/air.

The lowest mode in the band diagram for the unit cell in Fig. 2 is plotted in Fig. 3(a). For a given frequency, the group speed for waves propagating in the $x$-direction, $c_{g x}$, corresponds to the

${ }^{1}$ The term group speed is here used to refer to the scalar values $c_{g x}$ and $c_{g y}$. 
(a)

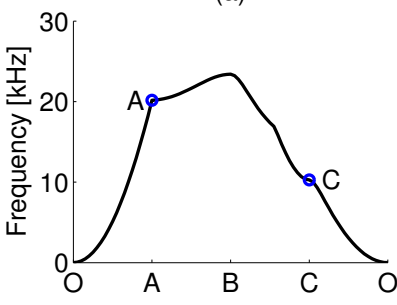

(b)

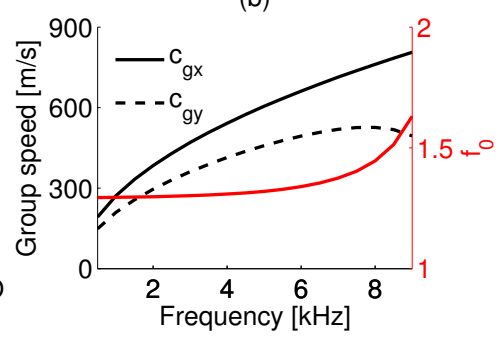

Figure 3: (a) The lowest mode for the unit cell in 2, and (b) the corresponding group speeds and objective function.

slope of the O-A part. While the group speed for waves propagating in the $y$-direction, $c_{g y}$, is the slope at the given frequency of the O-C part. In Fig. 3(b) the computed group speeds and their ratio, $f_{0}$, are plotted for frequencies up to $9 \mathrm{kHz}$. The ratio $f_{0}$ for the low frequency range has an almost constant value of approximately 1.4 up to $6.0 \mathrm{kHz}$, before it increases when inertia effects become important. With the given manufacturing constraints a ratio of just below 1.5 for low frequency waves is decent, and will be used to benchmark the optimized designs.

\subsection{Topology optimization}

Topology optimization is a method which iteratively updates the material distribution in a design domain based on element gradients. To compute the element gradients it is necessary to introduce a continuous interpolation between material (aluminum) and void (air) in each finite element. Here we use a simple linear interpolation, such that the material properties of an element are given as

$$
\begin{aligned}
& E_{e}=10^{-6} E+\varphi_{e} E\left(1-10^{-6}\right), \\
& \rho_{e}=10^{-6} \rho+\varphi_{e} \rho\left(1-10^{-6}\right),
\end{aligned}
$$

where $\varphi_{e} \in[0,1]$ is the element design variable. As can be seen, neither the stiffness nor the density are allowed to vanish completely, since this would make the problem ill-posed.

\subsection{Method 1: Only low frequencies}

The simple way to maximize $f_{0}$ is to maximize the ratio between the frequency at point $\mathrm{A}$ and $\mathrm{C}$ in Fig. 3(a). This would indirectly affect $f_{0}$ for the low frequency range, but does not involve a computation of the group velocity. Thus, instead of solving Eq. (3), one solves the smaller equation system given by

$$
\left(\mathbf{K}(\mathbf{k})-\omega^{2} \mathbf{M}\right) \mathbf{u}=\mathbf{0}
$$

where

$$
\mathbf{K}(\mathbf{k})=\mathbf{K}_{0}-i\left(k_{x} \mathbf{K}_{1}+k_{y} \mathbf{K}_{2}\right)+k_{x}^{2} \mathbf{K}_{3}+k_{x} k_{y} \mathbf{K}_{4}+k_{y}^{2} \mathbf{K}_{5},
$$

and, as earlier mentioned, the computations of the matrices $\mathbf{K}_{i}$ and $\mathbf{M}$ are detailed in Appendix $\mathbf{A}$.

Equation (10) is solved for $\omega$ at $\mathbf{k}_{A}=\left(\pi / l_{x}, 0\right)$ and $\mathbf{k}_{C}=\left(0, \pi / l_{y}\right)$, giving us $\omega_{A}$ and $\omega_{C}$. We choose the objective in the optimization problem to be

$$
\min _{\varphi}\left(\frac{\omega_{C}}{\omega_{A}}\right)
$$

In topology optimization, the sensitivities (gradients) are found using the adjoint method. The sensitivities of the objective can be computed as

$$
\frac{\mathrm{d}\left(\frac{\omega_{C}}{\omega_{A}}\right)}{\mathrm{d} \varphi_{e}}=\frac{\frac{\mathrm{d} \omega_{C}}{\mathrm{~d} \varphi_{e}} \omega_{A}-\frac{\mathrm{d} \omega_{A}}{\mathrm{~d} \varphi_{e}} \omega_{C}}{\omega_{A}^{2}}
$$

where the sensitivities of $\omega_{A}$ and $\omega_{C}$ are given from the sensitivities of the eigenvalues, which can be found in e.g. [18]. The expression for the sensitivities of $\omega_{A}$ can be found from

$$
\frac{\mathrm{d} \omega_{A}^{2}}{\mathrm{~d} \varphi_{e}}=\frac{\mathbf{v}^{T} \frac{\mathrm{d} \mathbf{K}}{\mathrm{d} \varphi_{e}} \mathbf{u}-\omega_{A}^{2} \mathbf{v}^{T} \frac{\mathrm{d} \mathbf{M}}{\mathrm{d} \varphi_{e}} \mathbf{u}}{\mathbf{v}^{T} \mathbf{M u}} .
$$

Here $\mathbf{v}$ is the complex conjugate of $\mathbf{u}$. The sensitivity of $\omega_{C}$ can be computed in the same way.

\subsection{Method 2: Also for higher frequencies}

Method 1 is computationally simple and cheap, but it only indirectly affects $f_{0}$. A more general approach can be formulated by computing $f_{0}$ from the group velocities at a given frequency $\omega$, and use the adjoint method to directly compute the gradient of $f_{0}$. One advantage of this approach is that it can also be used for higher frequencies. Furthermore, with this method a range of specific frequencies can be included in the optimization.

The computation of the sensitivities of $f_{0}$ is slightly more involved and requires the solution of an additional system of equations. Differentiating $f_{0}$ with respect to the element design variable, and taking $c_{g x}$ to mean $\left.c_{g x}\right|_{\theta=0}$ and $c_{g y}$ to mean $\left.c_{g y}\right|_{\theta=90}$, we get

$$
\frac{\mathrm{d} f_{0}}{\mathrm{~d} \varphi_{e}}=\operatorname{sign}\left(\frac{c_{g x}}{c_{g y}}\right)\left(\frac{\frac{\mathrm{d} c_{g x}}{\mathrm{~d} \varphi_{e}} c_{g y}-c_{g x} \frac{\mathrm{d} c_{g y}}{\mathrm{~d} \varphi_{e}}}{c_{g y}^{2}}\right),
$$

where the details of how to compute the sensitivity of the group velocity components are given in Appendix B.

At higher frequencies, so-called bandgaps or partial bandgaps, where no waves propagate for a given frequency range, can arise. However, if these occur for the frequencies of interest, $f_{0}$ is no longer well-defined. Our formulation is not aimed at obtaining bandgaps, which could be better done with e. g. the formulation in [19], but attailoring the group velocity profile. Therefore, we add the following constraint to avoid the formation of partial bandgaps:

$$
c_{g y} \geq c_{g}^{0}
$$

where $c_{g}^{0}$ is an a priori defined lower limit on the group speed in the $y$-direction, e.g. $100 \mathrm{~m} / \mathrm{s}$. Defining it as a constant limit instead of a fraction of $c_{g x}$ prevents the optimization from pulling both $c_{g y}$ and $c_{g x}$ down (with the risk of a bandgap forming). 


\subsection{Manufacturability}

Out of consideration for the manufacturing, we choose to work with a unit cell of $15 \mathrm{~mm}$ times $15 \mathrm{~mm}$. After removing material, the remaining aluminum has to be connected, and the minimum width of holes and structural members is $1.2 \mathrm{~mm}$ (for a thickness of $2 \mathrm{~mm}$ ). In order to avoid islands of unconnected material we apply a conductivity constraint. Furthermore, using a regularization technique (filtering of the design variables) is found to be beneficial to get rid of fast oscillations (small features) in the design field.

\subsubsection{Conductivity constraint}

For a periodic material a homogenized conductivity (a macroscopic conductivity), $\boldsymbol{\kappa}^{*}$, can be computed. In the low frequency case, the optimizer will try to make the vertical connections as thin as possible. To prevent this we apply a minimum constraint on the conductivity in the $y$-direction

$$
\kappa_{y}^{*} \geq f_{\kappa} \kappa,
$$

where $f_{\kappa}$ is the required fraction of the conductivity, $\kappa$, of the base material. Computing the homogenized connectivity implies introducing another material interpolation:

$$
\kappa_{e}=10^{-6} \kappa+\varphi_{e}^{2} \kappa\left(1-10^{-6}\right) .
$$

The element conductivity, $\kappa_{e}$, is interpolated using a quadratic scheme - in a simple way penalizing intermediate element design variables. The computation of the homogenized conductivity for a periodic material is discussed in detail in [20]. A stiffness constraint can be used instead of a constraint on the conductivity, but this would have been computationally more costly. A fraction $f_{\kappa}=0.15$ has been used for all optimizations, because it gives the unit cell a minimum width of approximately $1.2 \mathrm{~mm}$ when only one connecting rod forms.

A conductivity constraint is no guarantee that unconnected islands of material do not appear, but [21] and [19] used it with success when optimizing material structures for high loss, and the results presented in Section 3 indicate that it is also enough for the considered problem.

\subsubsection{Regularization}

Since we want the final design to be manufacturable, we use a regularization scheme in the optimization to avoid too small details in the design [9]. A good regularization scheme is to apply a filter to the design variables (see e. g. [22]), such as

$$
\tilde{\varphi}_{e}=\frac{\sum_{i \in N_{e}} w\left(\mathbf{x}_{i}\right) \varphi_{e}}{\sum_{i \in N_{e}} w\left(\mathbf{x}_{i}\right)},
$$

where

$$
w\left(\mathbf{x}_{i}\right)=R-\left\|\mathbf{x}_{i}-\mathbf{x}_{e}\right\|,
$$

is a linearly decaying weight function, $N_{e}$ is a list of elements for which the centers lie inside the filter radius $R$, and $\mathbf{x}_{i}$ is the spatial location of element center $i$. Note, when using a filter, the filtered design variable $\tilde{\varphi}_{e}$ is then substituted for $\varphi_{e}$ in all the previously presented equations.
The problem with the filtering scheme in Eq. (19), is that it enforces areas in the design with intermediate material properties. Since we want to be able to end up with designs that are physically realistic and realizible (where each element represents either no material or material), we apply a so-called threshold projection [23] on top of the filtering. We use a projection method similar to [24], which is basically a continuous step function thresholding element densities (the filtered design variables) at $\eta=0.5$. Here given as

$$
\bar{\varphi}_{e}=\frac{\tanh (\eta \beta)+\tanh \left(\left(\tilde{\varphi}_{e}-\eta\right) \beta\right)}{\tanh (\eta \beta)+\tanh ((1-\eta) \beta)},
$$

where $\eta$ is the threshold value, and $\beta$ is a parameter deciding the steepness of the step function.

The argument for doing this, is that we have observed using a large filter radius of $1.5 \mathrm{~mm}$ (5 elements), combined with projection, results in better designs (for the problems considered here) than gradually reducing the filter radius. We use a continuation scheme on the projection parameter, gradually increasing $\beta$ from 2 to 32 , to avoid getting stuck too early in a local minimum.

Remark, in all the previously mentioned formulas the design variable $\varphi_{e}$ should be substituted with the projected element density $\bar{\varphi}_{e}$, and the sensitivity with respect to the design variable is found using the chain rule

$$
\frac{\mathrm{d}}{\mathrm{d} \varphi_{e}}=\frac{\mathrm{d}}{\mathrm{d} \bar{\varphi}_{e}} \frac{\mathrm{d} \bar{\varphi}_{e}}{\mathrm{~d} \tilde{\varphi}_{e}} \frac{\mathrm{d} \tilde{\varphi}_{e}}{\mathrm{~d} \varphi_{e}},
$$

The complete optimization problem for method 1 is

$$
\begin{array}{ll} 
& \min _{\varphi}\left(\frac{\omega_{C}}{\omega_{A}}\right) \\
\text { s.t.: } & \text { Eq. } 10, \\
& \kappa_{y}^{*} \geq 0.15 \kappa, \\
& 0 \leq \varphi_{e} \leq 1 \quad e=1, \ldots, N,
\end{array}
$$

while for method 2 it is

$$
\begin{array}{cll}
\max _{\varphi}: & \min _{\omega}\left(f_{0}\right), \quad \omega \in\left[\omega_{1}, \omega_{2}\right] \\
\text { s.t.: } & \text { Eq. 3, } & \\
& \text { Eq. B.15, } & \\
& \kappa_{y}^{*} \geq 0.15 \kappa, & \\
& \kappa_{x}^{*} \geq 0.15 \kappa, & \\
& c_{g y}(\omega) \geq c_{g}^{0}, \quad \omega \in\left[\omega_{1}, \omega_{2}\right] \\
& 0 \leq \varphi_{e} \leq 1, \quad e=1, \ldots, N
\end{array}
$$

where the constraints on $\kappa_{x}$ and $c_{g y}$ only are necessary for higher frequencies. Something that will be discussed further in Section 3.

Topology optimization is used iteratively to decide if an element should contain material (aluminum) or not. In each iteration the nonlinear optimization algorithm MMA [25] is used to update the design variables in order to improve the objective while satisfying the constraints. All results presented in Section 3 are obtained within 300 iterations.

As a final note, it should be mentioned that since we are using projection the filtering does not guarantee a minimum 


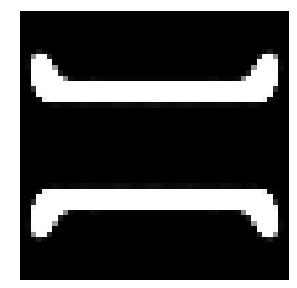

Figure 4: The unit cell resulting from optimizing with method 1. (a)

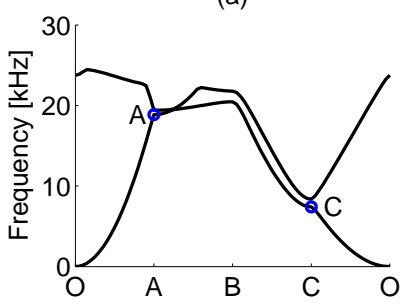

(b)

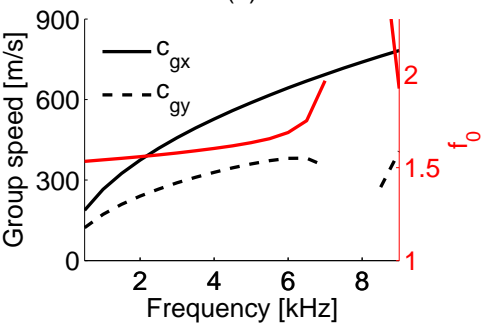

Figure 5: (a) The two lowest modes in the band diagram for the unit cell design in Fig.4, and (b) the corresponding performance $\left(f_{0}\right)$ and group speeds.

length scale. Furthermore, the optimization can still end up with a certain amount of intermediate material (neither solid nor void) when it is beneficial for the performance. This is not a problem for the low frequency designs. However for the high frequency designs it is a problem. One way to amend this will be shown in Section 3.3.

\section{Results}

The result section is divided into three subsections. First, designs obtained using method 1 are discussed. Thereafter, these will be compared to the results obtained using method 2 . Finally, the results obtained when applying method 2 to higher frequencies are discussed.

Remark, when computing the performance of the optimized designs we model all elements where $\varphi<0.5$ as void and all elements with $\varphi \geq 0.5$ as solid. The square unit cells are discretized with 50 by 50 Mindlin elements with selective reduced integration of the shear deformation terms.

\subsection{Method 1}

\subsubsection{Comparison to benchmark design}

Applying method 1 to maximize the directionality of the low frequency waves results in the design shown in Fig. 4. It is slightly different from the intuitive benchmark design, with two long slits instead of one, and with a small bend at both ends of the slit. Note that a double symmetry has been imposed in the optimization.

Remark, in the design coming out of the topology optimization there is a slight amount of intermediate densities (shown as gray pixels in the unit cell plot). However, when evaluating the performance all elements with a density above 0.5 are considered to be aluminum and those below are modeled as void. When the amount of gray is small, as in this design, it does not

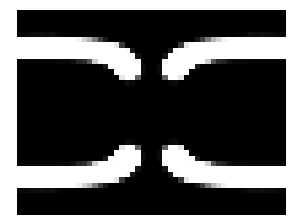

Figure 6: The unit cell resulting from optimizing with method 1 using a rectangular domain. (a)

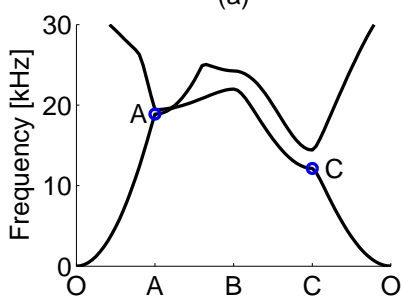

(b)

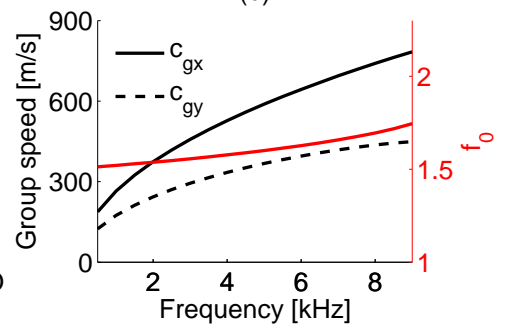

Figure 7: (a) The two lowest modes in the band diagram for the unit cell design in Fig. 6, and (b) the corresponding performance $\left(f_{0}\right)$ and group speeds.

affect the performance. The performance of the design is shown in Fig. 5.

The performance is clearly better than the intuitive slit design, with an $f_{0}$ of approximately 1.6 up to around $6 \mathrm{kHz}$. Around $7 \mathrm{kHz}$ the performance curve has a discontinuity, which can be best understood by considering Fig. 5(a), where the two first modes in the band diagram are plotted. Point $\mathrm{C}$ has a tiny partial bandgap starting just before $\omega=7.5 \mathrm{kHz}$, and the performance curve is based on an evaluation of group velocities at $0.5 \mathrm{kHz}$ intervals. At 7.5 and $8.0 \mathrm{kHz}$ the group velocity in the $y$-direction is undefined, and it is first defined again at $8.5 \mathrm{kHz}$ (where $c_{g x}$ is computed based on the second band).

\subsubsection{Rectangular unit cell}

The design in Fig. 4 contains two slits, as opposed to one for the intuitive guess. Thus, it is natural to ask if a non-square unit cell would be beneficial. Therefore, we investigate whether an even better performance can be obtained with a rectangular unit cell. Figure 6 shows an optimized rectangular unit cell with a height to width ratio of approximately $3 / 4$ (the height is decreased to $11.4 \mathrm{~mm}$ ).

The resulting performance plot can be seen in Fig. 7. When considering the corresponding band diagram, it is important to keep in mind that the unit cell is rectangular, such that the distance $\mathrm{O}-\mathrm{C}$ is shorter than $\mathrm{O}-\mathrm{A}$. This means that the vertical distance between point $\mathrm{A}$ and $\mathrm{C}$ is not directly comparable to the band diagrams for the square designs.

The performance of the rectangular unit cell is approximately the same as the square cell, and so is the topology. Based on this result, there is no immediate reason to believe that the rectangular unit cell will provide an improved performance. Again, note that the frequency at point $\mathrm{C}$ in Fig. 7(a) is higher than in Fig. 5(a), because the unit cell height is smaller. 


\subsubsection{Non-symmetric design}

The symmetry in the previously presented designs have been imposed by a vertical and horizontal symmetry line in the optimization. Therefore, it is natural to investigate if a better performance can be achieved by removing these. It is important to point out that plotting the first mode by traversing the line $\mathrm{O}$ A-B-C-O is normally only done for designs containing the two symmetry lines. However, we stick with the same performance measure, because if the added freedom is of importance then it should improve. The resulting design is shown in Fig. 8, with the corresponding performance plot in Fig. 9.

The topology of the non-symmetric design is very similar to the symmetric, but with one of the void slitsdisplaced slightly. The performance is about the same, but $f_{0}$ starts to increase slightly earlier, because point $\mathrm{C}$ is pushed a bit lower. We believe this effect can be explained by the increased curvature of the slits.

\subsection{Method 2 - low frequencies}

\subsubsection{Comparison to method 1}

With method 2 one can choose to maximize $f_{0}$ for one or several frequencies. Since the results from method 1 revealed that $f_{0}$ is almost constant (or slightly increasing) up to approximately $6 \mathrm{kHz}$, we gather that for the low frequency range it does not matter whether we optimize for one or several frequencies. Thus, since it is computationally more expensive to optimize for more frequencies, only one frequency is included in the low frequency optimizations.

The unit cell in Fig. 10(a) has been achieved by maximizing $f_{0}$ at $3.0 \mathrm{kHz}$. The topology is very similar to the symmetric design obtained with method 1, but with bi-directional bends at the end of the slits. The corresponding performance, shown in Fig. 11, is almost identical. However, it can be seen that the performance curve flatter (almost constant) for a larger range of frequencies.

\subsubsection{Increasing the frequency}

The result in Fig. 10(a) has been achieved by maximizing $f_{0}$ at $3.0 \mathrm{kHz}$. Optimizing for a lower or higher frequency does not influence the design noticeably - until we reach $5 \mathrm{kHz}$. As can be seen in Fig. 10(b), where the optimized design for $\omega=5.0$ $\mathrm{kHz}$ is shown, the design now contains the same kind of bends as the one obtained with method 1 . The performance, shown in Fig. 12, is slightly improved at $5.0 \mathrm{kHz}$ versus the design in Fig. 10(a).

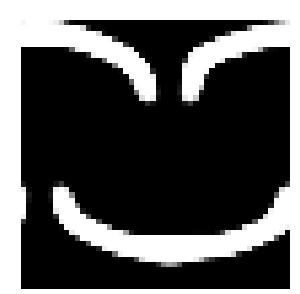

Figure 8: The unit cell resulting from optimizing with method 1 without symmetry constraints. (a)
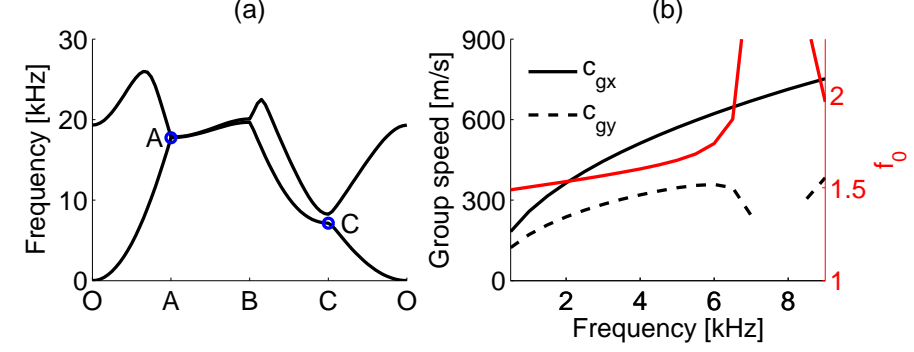

Figure 9: (a) The two lowest modes in the band diagram for the unsymmetric unit cell design in Fig. 6, and (b) the corresponding performance $\left(f_{0}\right)$ and group speeds.

Now, if the frequency is increased further, a circular inclusion clearly forms. Already at $5.5 \mathrm{kHz}$ the resulting topology is quite different (see Fig. 10 (c)). It looks like the optimization tries to form a resonant inclusion very similar to the one used in [12]. Actually, the rods connecting the inclusion in the $x$-direction are low density elements (soft material), and disappear after thresholding (making the structure unphysical). In order to amend this, the optimization at $5.5 \mathrm{kHz}$ is rerun with an additional conductivity constraint in the $x$-direction (same as in the $y$-direction). Even though this is not a guaranteed solution, it prevents the closing of the circle, because the isolated island of material would be so large that the design would not fulfill the conductivity constraint. The optimized design is shown in Fig. 10(d), and the performance is plotted in Fig. 13.

The performance of the design in Fig. 10(d) at the lowest frequencies is not as good as the lower frequency designs. However, it has a very good performance at the higher frequencies, until it reaches the first partial bandgap (where $c_{g y}$ is undefined).

\subsection{Method 2 - high frequencies}

Low frequency wave propagation is to a high degree determined by the stiffness, but as seen in the previous section the

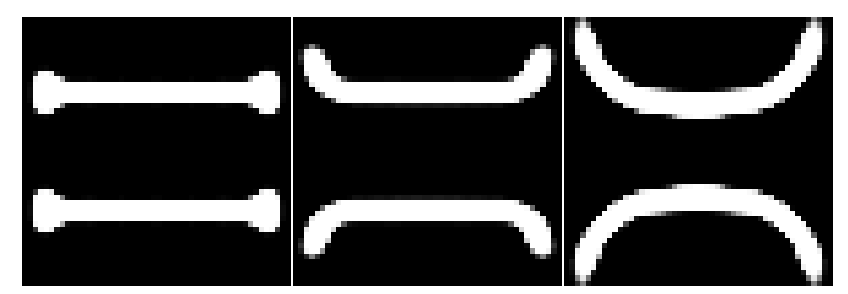

(a)

(b)

(c)

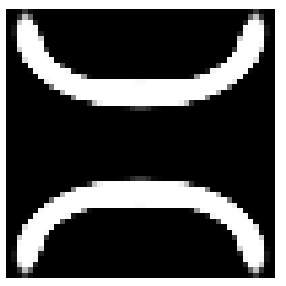

(d)

Figure 10: Unit cells from optimization of $f_{0}$ at (a) $3.0 \mathrm{kHz}$, (b) $5.0 \mathrm{kHz}$, (c) $5.5 \mathrm{kHz}$, and (d) $5.5 \mathrm{kHz}$ with additional conductivity constraint in $x$-direction 
(a)

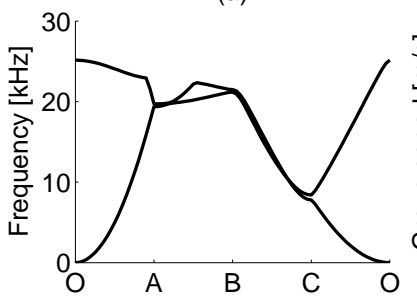

(b)

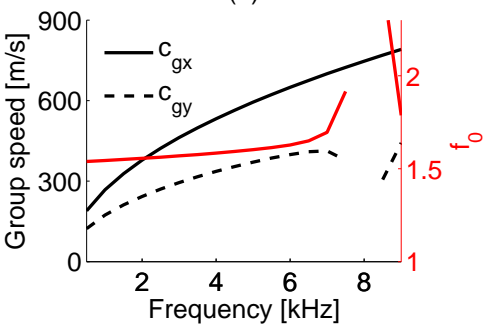

Figure 11: (a) The two lowest modes in the band diagram for the unit cell design in Fig.10(a) optimized at $3.0 \mathrm{kHz}$, and (b) the corresponding performance $\left(f_{0}\right)$ and group speeds.

(a)

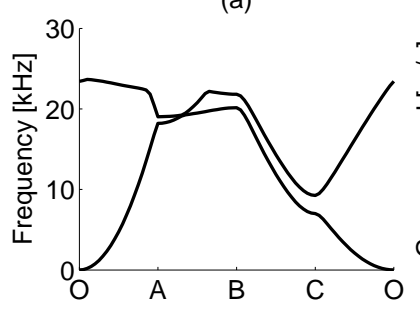

(b)

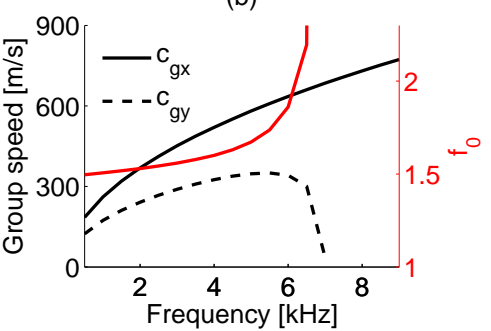

Figure 12: (a) The two lowest modes in the band diagram for the unit cell design in Fig. 10(b) optimized at $5.0 \mathrm{kHz}$, and (b) the corresponding performance $\left(f_{0}\right)$ and group speeds.

design changes when the frequency is increased and inertia effects start to play a part. In this section we want to show how method 2 can be applied to create optimized designs for frequencies above the first mode.

\subsubsection{One frequency}

For higher frequencies two additional constraints are active in the optimization, both of which are included in Eq. (24). Firstly, for higher frequencies we chose to apply a conductivity constraint in both directions. Unless this is done, designs with a lot of intermediate density or unconnected islands of material form. Secondly, a constraint on the minimum group speed $c_{g x}$ is applied to prevent the optimizer from creating a design with a partial bandgap. For the frequencies considered in the following, we found $c_{g}^{0}=500[\mathrm{~m} / \mathrm{s}]$ to work well.

Even with these constraints, which are helpful, the opti-

(a)

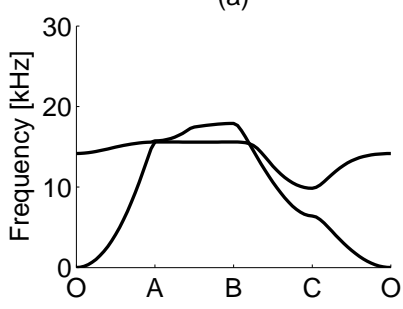

(b)

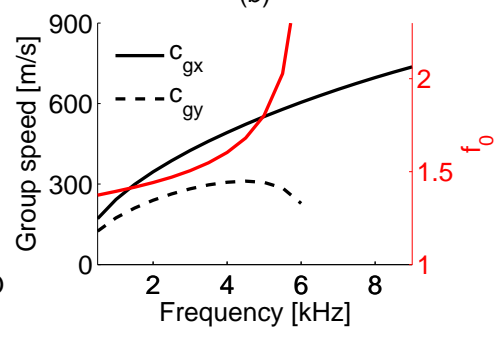

Figure 13: (a) The two lowest modes in the band diagram for the unit cell design in Fig. 10(d) optimized at $5.5 \mathrm{kHz}$ with additional conductivity constraint, and (b) the corresponding performance $\left(f_{0}\right)$ and group speeds.

mized designs are not ready for manufacturing. Figures 14(a) and (b) show designs where $f_{0}$ has been optimized for a single frequency - 25 and $30 \mathrm{kHz}$, respectively. Both designs show some amount of intermediate density, and both have, from a manufacturing point of view, too thin features.

The problem with the intermediate densities is that the optimizer uses the interpolation between aluminum and void to create a better performing design by including intermediate densities, which cannot be manufactured. When we threshold the designs to evaluate the performance the result is unpredictable. In most cases the performance will deteriorate drastically. For example the performance, $f_{0}$, of the $25 \mathrm{kHz}$ design, shown together with a portion of the band diagram in Fig. 15, deteriorates to below 1.3 (as opposed to an $f_{0}=2.3$ for the non-thresholded design). The performance deterioration of the $30 \mathrm{kHz}$ design is even worse (see Fig. 16), because the behavior of the thresholded design is very different from the non-thresholded design (the thin bars at the top and bottom are not connected across unit cells). In Fig. 16(b) $c_{g x}$ is not plotted below $27 \mathrm{kHz}$, because, as can be seen from Fig. 16(a), there is a partial bandgap in the $x$-direction. For the same reason, neither $c_{g x}$ nor $c_{g y}$ are defined above $32 \mathrm{kHz}$.

\subsubsection{Frequency range}

The discussed issues do not, unfortunately, disappear when more frequencies are included. Figure 17 shows a design where $f_{0}$ is optimized at the frequencies $27.5,30.0$, and $32.5 \mathrm{kHz}$, simultaneously. The issues are still there, and the performance of the thresholded design is again unsatisfactory. The performance is plotted in Fig. 18 together with a band diagram, where dashed lines indicate the three frequencies included in the optimization. When including the interpolation in the evaluation of $f_{0}$, the ratio is above 1.8 , which is quite good. However, the performance of the thresholded design, which is shown in Fig. 18 is far below 1 for the whole frequency range of interest $(27.5-32.5 \mathrm{kHz})$, for the same reason that the $30.0 \mathrm{kHz}$ design

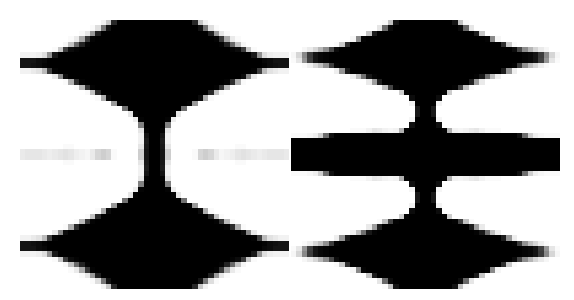

(a)

(b)

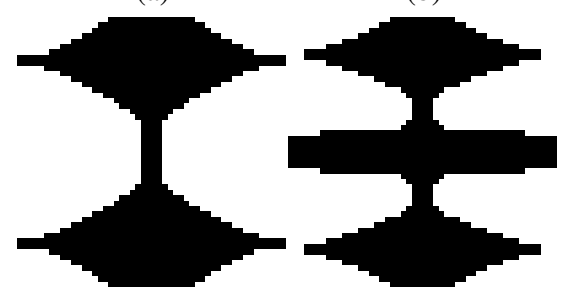

(c)

(d)

Figure 14: Unit cells from optimization of $f_{0}$ at (a) $25 \mathrm{kHz}$, (b) $30 \mathrm{kHz}$. (c) and (d) show the corresponding thresholded versions. 
(a)
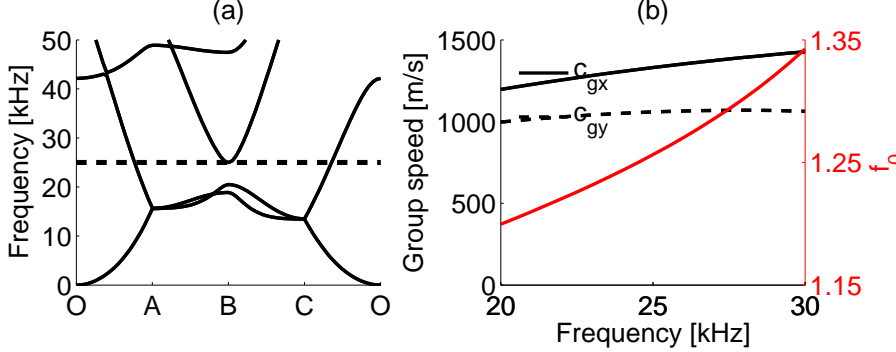

Figure 15: (a) The band diagram up to $50 \mathrm{kHz}$ for the unit cell in Fig.10(c) optimized at $25.0 \mathrm{kHz}$ (dashed line), and (b) the corresponding performance $\left(f_{0}\right)$ and group speeds. (a)

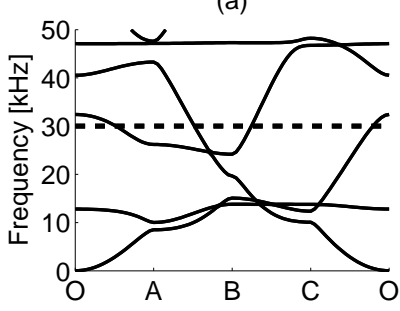

(b)

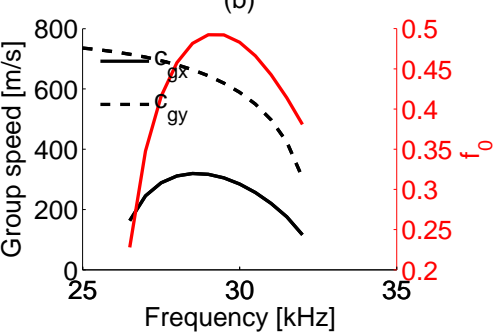

Figure 16: (a) The band diagram up to $50 \mathrm{kHz}$ for the unit cell in Fig.10(d) optimized at $30.0 \mathrm{kHz}$ (dashed line), and (b) the corresponding performance $\left(f_{0}\right)$ and group speeds.

in the previous section performed poorly - it behaves radically different than the non-thresholded design.

\subsubsection{Manufacturable design}

The robust optimization approach proposed in [26] should be ideally suited to amend the problems discussed above. Instead of optimizing the performance of a single design, we optimize the performance for three versions of the design. In addition to the blueprint design $(\eta=0.5$ in Eq. (21)), we optimize one eroded (corresponding to a threshold value $\eta_{e}>0.5$ in Eq. (21)), and one dilated (corresponding to a threshold value $\eta_{d}<0.5$ in Eq. 21) design.

Running such an optimization for the frequency range (27.5$32.5 \mathrm{kHz}$ with $\eta_{e}=0.65$ and $\eta_{d}=0.35$, we obtain the blueprint design in Fig. 19. There is only a small amount of intermediate densities in the design, and, due to the robustness to dilation and erosion, the performance of the thresholded design (shown in Fig. 20) is good. Furthermore, the design now fulfills the manufacturing constraints.

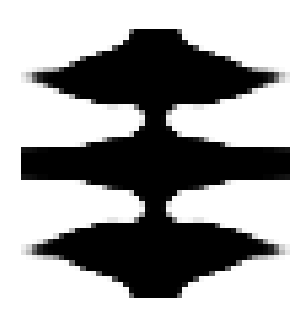

Figure 17: The unit cell resulting from optimizing for a range the frequencies $27.5,30.0$, and $32.5 \mathrm{kHz}$. (a)
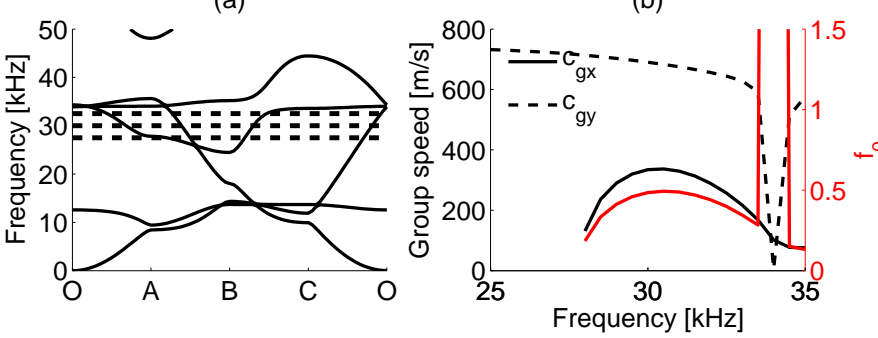

Figure 18: (a) The band diagram up to $50 \mathrm{kHz}$ for the unit cell in Fig. 17 (the thresholded version of it) optimized at $27.5-32.5 \mathrm{kHz}$ (dashed lines indicate frequencies included in the optimization), and (b) the corresponding performance $\left(f_{0}\right)$ and group speeds. Remark $f_{0}$ is not plotted for frequencies where $c_{g x}$ or $c_{g y}$ are undefined.

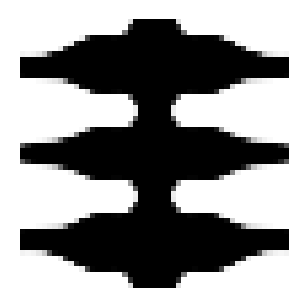

Figure 19: Unit cell of blueprint design resulting from a robust optimization for the frequencies $27.5,30.0$, and $32.5 \mathrm{kHz}$.

From Fig. 20 it can be seen that within the considered frequency range $f_{0}$ is lowest at $27.5 \mathrm{kHz}$, but even there $f_{0}$ is still above 1.8 and the ratio increases slightly towards $32.5 \mathrm{kHz}$. The group velocity profile at $30.0 \mathrm{kHz}$ for the optimized robust design is plotted in Fig. 21 - illustrating how the energy would propagate about twice as fast in the horizontal direction.

\section{Experimental verification}

\subsection{Set-up}

A water-jetting technique is used to cut a 21 by 21 unit cell array of the optimized design in Fig. 19. The final design is cut from a $457 \mathrm{~mm}$ by $457 \mathrm{~mm}$ by $2 \mathrm{~mm}$ aluminum plate (Young's modulus $E=69 \mathrm{GPa}$, Poisson's ratio $v=0.33$, and the density $\rho=2700 \mathrm{~kg} / \mathrm{m}^{3}$ ). One unit cell is left uncut at the center of the plate so that a piezoelectric (PZT) disc can be attached (a)

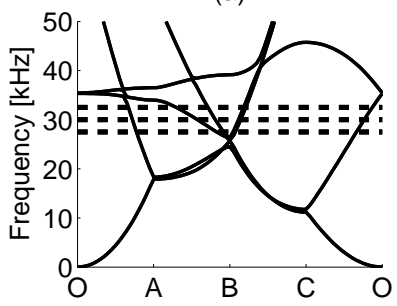

(b)

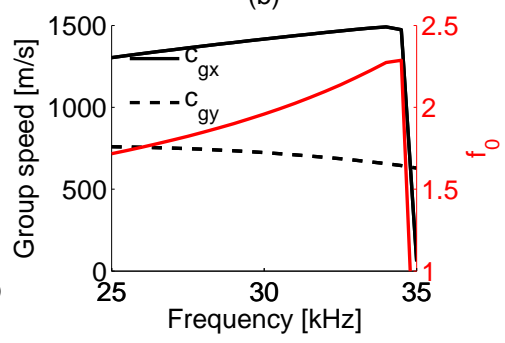

Figure 20: (a) The band diagram up to $50 \mathrm{kHz}$ for the unit cell in Fig.19 (as always the thresholded version) optimized at $27.5-32.5 \mathrm{kHz}$ (dashed lines indicate frequencies included in the optimization), and (b) the corresponding performance $\left(f_{0}\right)$ and group speeds. 


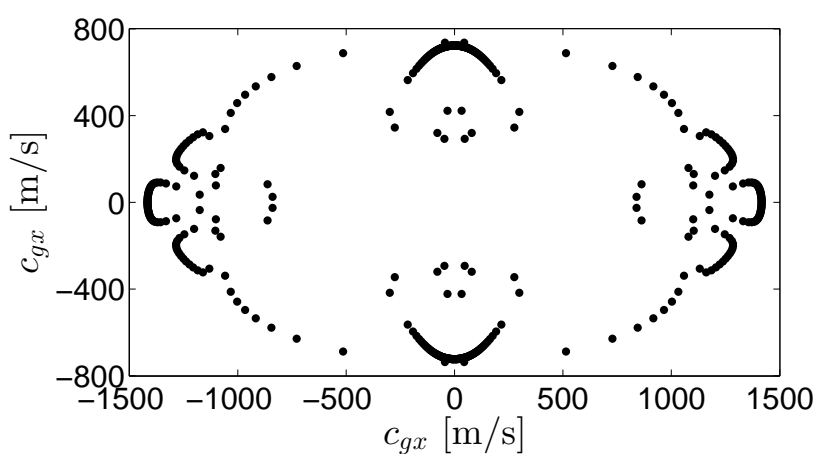

Figure 21: The group velocity profile at $30.0 \mathrm{kHz}$ for the unit cell in Fig. 19. Each point corresponds to the group velocity (or velocities) at a discrete angle.

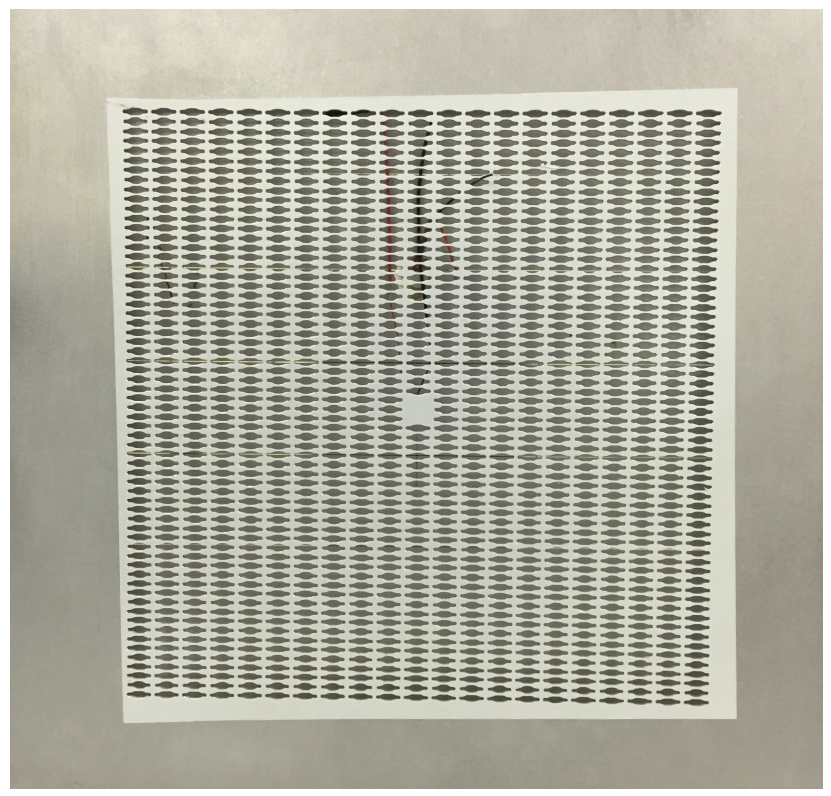

Figure 22: A picture of the perforated aluminum plate with reflective tape attached.

to apply the desired excitation to the plate. The resulting plate and a more detailed view of a unit cell are shown in Fig. 22 and 23 , respectively. A layer of reflective tape is applied to the solid material to reduce the noise in the SLV measurements. Then, a measurement grid of 150 by 150 equally spaced points is overlaid onto the plate. Points that land on the void of the plate are manually removed to account for any discrepancies. Ten measurements are taken at each point and then averagedto reduce noise. A PZT disc of $5 \mathrm{~mm}$ diameter and a resonance frequency of up to $450 \mathrm{kHz}$ from Steminc Corp. is chosen for our experiment. The PZT disc is used to excite the plate using a seven cycle Hanning modulated sinusoid burst at a center frequency of $30 \mathrm{kHz}$. The resulting out-of-plane velocity at each grid point is recorded through the SLV software.

\subsection{Results}

The SLV measurements show the expected anisotropic wave propagation behavior, which is clear from the two snapshots of the experimental wave field shown in Fig. 24, in which the theoretical group velocity profile from Fig. 21 is also plotted.

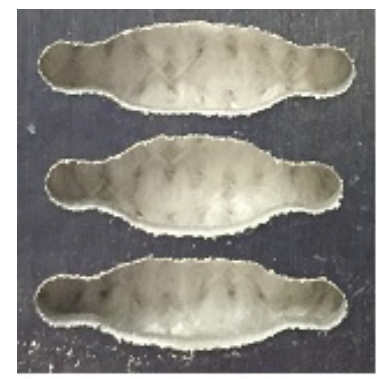

Figure 23: A close-up picture of one unit cell in the perforated aluminum plate.

Compared to the results in [12], the experimental measurements contain somewhat more noise. This can be explained by the smallest feature in the plate being almost halved compared to the plate in [12], making it difficult to accurately position the SLV on the smallest features (the thin horizontal bars). Thus, the noise is most pronounced in the area close to the center of the thin horizontal bars in the design. However, it is still

(a)

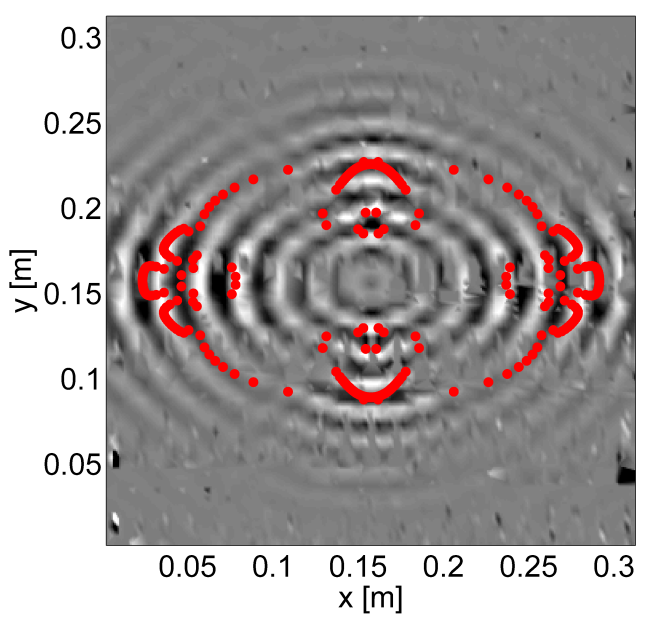

(b)

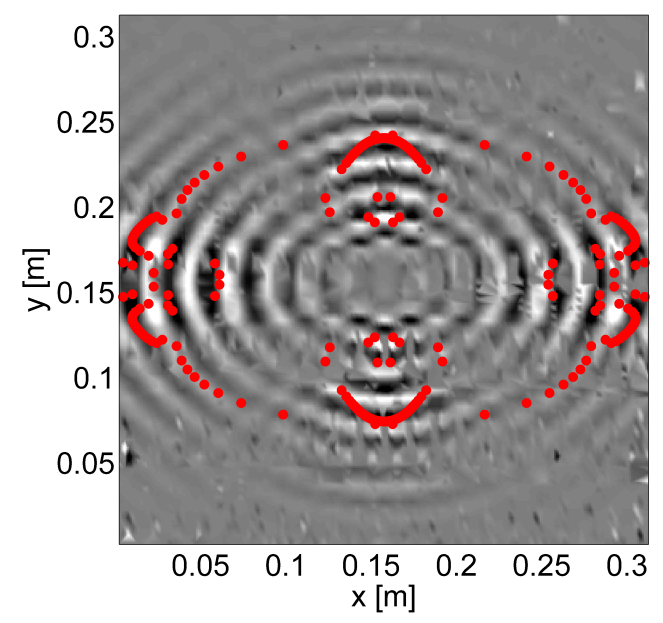

Figure 24: Snapshots of the experimental wave field overlaid with the theoretically computed group velocity profile from Fig. 21 (red dots). (a) $t=195 \mu \mathrm{s}$, and (b) $t=215 \mu \mathrm{s}$. 
clear from the measurements that the expected directional wave propagation profile is observed.

Finally, a 2D Fourier transform of the experimental wave field at $30 \mathrm{kHz}$ is included in Fig. 25, which illustrates the plate dispersion in the wave number domain.

\section{Conclusion}

In this work we have presented a topology optimization method for creating periodically perforated plates with tailored directional wave propagation characteristics. The method is based on a gradient-based algorithm where we aim to find optimized distributions of material and air in a computational unit cell. As optimization objective we choose to maximize the ratio of group velocities in two perpendicular directions.

Two methods to design plates with specific group velocity profiles for elastic waves have been presented. A simple efficient method for low frequency applications is based on a twopoint procedure where the difference between wave frequencies for two target wavenumbers is maximized. In this way it is indirectly ensured that the ratio between the group velocity in the two perpendicular directions is maximized. The method works only when targeting the lowest branch in the band diagram, however, a good efficiency is obtained since a direct computation of the group velocity is avoided. Examples show that the method works well in the low frequency case (corresponding to the lowest branch in the band diagram).

The second proposed method is more computationally involved and is based on a direct computation of the group velocity - however, as opposed to the first method, this method is generally applicable and can be applied to target both low and higher-order branches in the band diagram. In order to ensure a well-defined ratio of group velocities in the optimization process we have shown that it is necessary to enforce a constraint on the minimum group velocity in order avoid the formation of partial bandgaps. As these are characterized by a vanishing

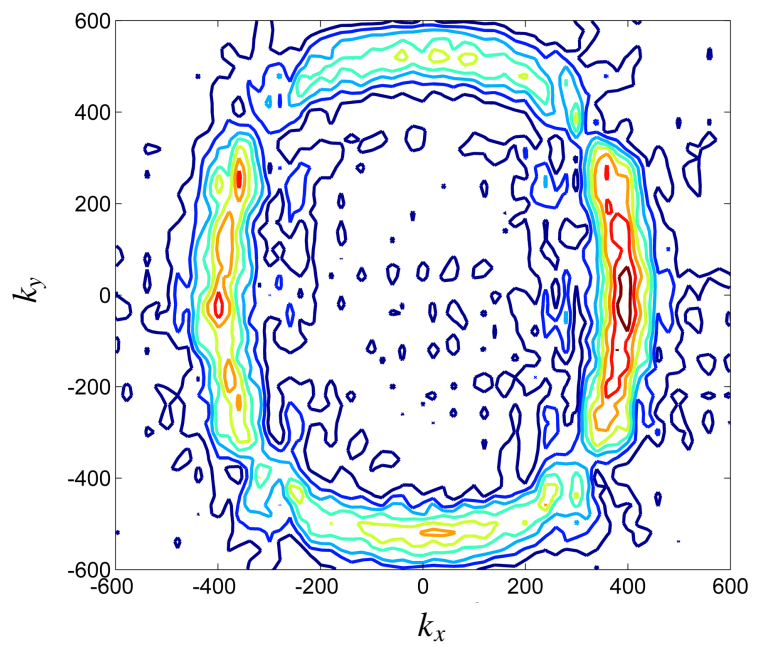

Figure 25: Experimental 2D FT (when excited at $30 \mathrm{kHz}$ ) for representation of plate dispersion in the $k_{x}-k_{y}$ domain. group velocity this would render the group velocity ratio undefined. By including this constraint and also employing a robust optimization formulation we have successfully designed a perforated structure with a group velocity ratio exceeding 1.8 in a frequency range from $27.5 \mathrm{kHz}$ to $32.5 \mathrm{kHz}$.

A sample plate was fabricated with optimized perforations created using a water-jetting technique. Scanning laser vibrometer measurements on the optimized plate indicate good agreement with the numerically predicted directional behavior. Thus, the proposed method appears to be promising for enabling an enhanced control of wave propagation anisotropy in mechanical structures. In the presented example a mild anisotropy is obtained, however, in theory, stronger anisotropy could possibly be obtained by targeting higher branches in the band diagram. The main challenge appears to be to ensure manufacturability of the optimized designs. Although here applied specifically to perforated plate structures, the methodology is quite general and can easily be adapted to, for instance, bulk elastic waves and/or multi-material structures where the manufacturability requirements could be less restrictive.

\section{Acknowledgement}

For this work E. Andreassen and J. S. Jensen received funding from the Danish Research Agency through the innovation consortium $\mathrm{F} \bullet \mathrm{MAT}$.

\section{Appendix A. System matrices}

To find the system matrices for the eigenvalue problem

$$
\left(\mathbf{K}_{k}(\theta, \omega)-k \mathbf{M}_{k}(\theta)\right) \mathbf{u}_{k}=\mathbf{0},
$$

we consider the governing plate equations, as given in [27]:

$$
\begin{gathered}
\boldsymbol{\nabla}^{T} \mathbf{G}(\boldsymbol{\nabla} w-\boldsymbol{\Theta})=\rho h \frac{\partial^{2} w}{\partial t^{2}}, \\
\boldsymbol{\partial}^{T} \mathbf{D} \partial \boldsymbol{\Theta}+\mathbf{G}(\boldsymbol{\nabla} w-\boldsymbol{\Theta})=\frac{\rho h^{3}}{12} \frac{\partial^{2} \boldsymbol{\Theta}}{\partial t^{2}},
\end{gathered}
$$

where $w(\mathbf{x}, t)$ denotes the out-of-plane deflection of a point of the mid-surface at location $\mathbf{x}=[x, y]^{T}, \boldsymbol{\Theta}=\left[\theta_{x}, \theta_{y}\right]^{T}$ is a vector containing the two rotations of a material line normal to the mid-surface about the two in-plane axes, $\rho$ is the material density, while $h$ is the plate thickness. Also, $\mathbf{D}=\mathbf{D}(\mathbf{x})$ and $\mathbf{G}=\mathbf{G}(\mathbf{x})$ are the plate constitutive matrices. At a given point in the plate material, which is locally isotropic, these are:

$$
\mathbf{D}=\frac{E h^{3}}{12\left(1-v^{2}\right)}\left[\begin{array}{ccc}
1 & v & 0 \\
v & 1 & 0 \\
0 & 0 & \frac{(1-v)}{2}
\end{array}\right],
$$

and

$$
\mathbf{G}=\frac{E}{2(1+v)}\left[\begin{array}{cc}
\frac{5}{6} h & 0 \\
0 & \frac{5}{6} h
\end{array}\right]
$$


where $E$ is the elasticity module of the material, and $v$ is Poisson's ratio.

Finally, the differential operator $\boldsymbol{\partial}$ is defined as:

$$
\boldsymbol{\partial}=\left[\begin{array}{ll}
1 & 0 \\
0 & 0 \\
0 & 1
\end{array}\right] \frac{\partial}{\partial x}+\left[\begin{array}{ll}
0 & 0 \\
0 & 1 \\
1 & 0
\end{array}\right] \frac{\partial}{\partial y}=\alpha_{1} \frac{\partial}{\partial x}+\alpha_{2} \frac{\partial}{\partial y}
$$

After applying the Bloch theorem and discretizing we end up with the system matrices

$$
\begin{aligned}
\mathbf{K}_{k}(\theta, \omega) & =\left[\begin{array}{cc}
-i \mathbf{A}_{1} & \mathbf{A}_{0}-\omega^{2} \mathbf{M} \\
\mathbf{I} & \mathbf{0}
\end{array}\right], \\
\mathbf{M}_{k}(\theta) & =\left[\begin{array}{cc}
-\mathbf{A}_{2} & \mathbf{0} \\
\mathbf{0} & \mathbf{I}
\end{array}\right]
\end{aligned}
$$

where the three submatrices are given as sums over element matrices

$$
\begin{aligned}
& \mathbf{A}_{0}=\sum_{e} \mathbf{k}_{0}^{e}, \\
& \mathbf{A}_{1}=\sum_{e}\left(\cos (\theta) \mathbf{k}_{1}^{e}+\sin (\theta) \mathbf{k}_{2}^{e}\right), \\
& \mathbf{A}_{2}=\sum_{e}\left(\cos ^{2}(\theta) \mathbf{k}_{3}^{e}+\cos (\theta) \sin (\theta) \mathbf{k}_{4}^{e}+\sin ^{2}(\theta) \mathbf{k}_{5}^{e}\right) .
\end{aligned}
$$

With the nodal displacements interpolated over the element using the shape function matrices $\mathbf{N}_{w}$ and $\mathbf{N}_{\Theta}$, as follows

$$
\mathbf{u}^{e}=\left[\begin{array}{cc}
\mathbf{N}_{w}(x, y) & \mathbf{0} \\
\mathbf{0} & \mathbf{N}_{\boldsymbol{\Theta}}(x, y)
\end{array}\right]\left[\begin{array}{c}
\tilde{\mathbf{w}}^{e} \\
\tilde{\mathbf{\Theta}}^{e}
\end{array}\right]
$$

The corresponding expressions for the element matrices are

$$
\begin{aligned}
& \mathbf{k}_{0}^{e}=\int_{\Omega^{e}}\left[\begin{array}{cc}
-\boldsymbol{\nabla} \mathbf{N}_{w}^{T} \mathbf{G} \boldsymbol{\nabla} \mathbf{N}_{w} & \left(\mathbf{N}_{\boldsymbol{\Theta}}^{T} \mathbf{G} \boldsymbol{\nabla} \mathbf{N}_{w}\right)^{T} \\
\mathbf{N}_{\boldsymbol{\Theta}}^{T} \mathbf{G} \boldsymbol{\nabla} \mathbf{N}_{w} & -\boldsymbol{\partial} \mathbf{N}_{\boldsymbol{\Theta}}^{T} \mathbf{D} \boldsymbol{\partial} \mathbf{N}_{\boldsymbol{\Theta}}-\mathbf{N}_{\boldsymbol{\Theta}}^{T} \mathbf{G} \mathbf{N}_{\boldsymbol{\Theta}}
\end{array}\right] d \Omega^{e}, \\
& \mathbf{k}_{1}^{e}=-\int_{\Omega^{e}}\left[\begin{array}{cc}
\mathbf{N}_{w}^{T} \boldsymbol{\beta}_{1}^{T} \mathbf{G} \boldsymbol{\nabla} \mathbf{N}_{w}-\boldsymbol{\nabla} \mathbf{N}_{w}^{T} \mathbf{G} \boldsymbol{\beta}_{1} \mathbf{N}_{w} & -\left(\mathbf{N}_{\Theta}^{T} \mathbf{G} \boldsymbol{\beta}_{1} \mathbf{N}_{w}\right)^{T} \\
\mathbf{N}_{\boldsymbol{\Theta}}^{T} \mathbf{G} \boldsymbol{\beta}_{1} \mathbf{N}_{w} & \mathbf{N}_{\boldsymbol{\Theta}}^{T} \boldsymbol{\alpha}_{1}^{T} \mathbf{D} \boldsymbol{\partial} \mathbf{N}_{\boldsymbol{\Theta}}-\boldsymbol{\partial} \mathbf{N}_{\boldsymbol{\Theta}}^{T} \mathbf{D} \boldsymbol{\alpha}_{1} \mathbf{N}_{\boldsymbol{\Theta}}
\end{array}\right] d \Omega^{e}, \\
& \mathbf{k}_{2}^{e}=-\int_{\Omega^{e}}\left[\begin{array}{cc}
\mathbf{N}_{w}^{T} \boldsymbol{\beta}_{2}^{T} \mathbf{G} \boldsymbol{\mathbf { V }} \mathbf{N}_{w}-\boldsymbol{\nabla} \mathbf{N}_{w}^{T} \mathbf{G} \boldsymbol{\beta}_{2} \mathbf{N}_{w} & -\left(\mathbf{N}_{\Theta}^{T} \mathbf{G} \boldsymbol{\beta}_{2} \mathbf{N}_{w}\right)^{T} \\
\mathbf{N}_{\Theta}^{T} \mathbf{G} \boldsymbol{\beta}_{2} \mathbf{N}_{w} & \mathbf{N}_{\Theta}^{T} \boldsymbol{\alpha}_{2}^{T} \mathbf{D} \boldsymbol{\partial} \mathbf{N}_{\Theta}-\boldsymbol{\partial} \mathbf{N}_{\Theta}^{T} \mathbf{D} \boldsymbol{\alpha}_{2} \mathbf{N}_{\Theta}
\end{array}\right] d \Omega^{e}, \\
& \mathbf{k}_{3}^{e}=-\int_{\Omega^{e}}\left[\begin{array}{cc}
\mathbf{N}_{w}^{T} \boldsymbol{\beta}_{1}^{T} \mathbf{G} \boldsymbol{\beta}_{1} \mathbf{N}_{w} & \mathbf{0} \\
\mathbf{0} & \mathbf{N}_{\boldsymbol{\Theta}}^{T} \boldsymbol{\alpha}_{1}^{T} \mathbf{D} \boldsymbol{\alpha}_{1} \mathbf{N}_{\boldsymbol{\Theta}}
\end{array}\right] d \Omega^{e}, \\
& \mathbf{k}_{4}^{e}=-\int_{\Omega^{e}}\left[\begin{array}{cc}
\mathbf{N}_{w}^{T}\left(\boldsymbol{\beta}_{1}+\boldsymbol{\beta}_{2}\right)^{T} \mathbf{G}\left(\boldsymbol{\beta}_{1}+\boldsymbol{\beta}_{2}\right) \mathbf{N}_{w} & \mathbf{0} \\
\mathbf{0} & \mathbf{N}_{\boldsymbol{\Theta}}^{T}\left(\boldsymbol{\alpha}_{1}+\boldsymbol{\alpha}_{2}\right)^{T} \mathbf{D}\left(\boldsymbol{\alpha}_{1}+\boldsymbol{\alpha}_{2}\right) \mathbf{N}_{\boldsymbol{\Theta}}
\end{array}\right] d \Omega^{e} \\
& \mathbf{k}_{5}^{e}=-\int_{\Omega^{e}}\left[\begin{array}{cc}
\mathbf{N}_{w}^{T} \boldsymbol{\beta}_{2}^{T} \mathbf{G} \boldsymbol{\beta}_{2} \mathbf{N}_{w} & \mathbf{0} \\
\mathbf{0} & \mathbf{N}_{\boldsymbol{\Theta}}^{T} \boldsymbol{\alpha}_{2}^{T} \mathbf{D} \boldsymbol{\alpha}_{2} \mathbf{N}_{\Theta}
\end{array}\right] d \Omega^{e} \\
& \mathbf{m}^{e}=-\int_{\Omega^{e}}\left[\begin{array}{cc}
\rho h \mathbf{N}_{w}^{T} \mathbf{N}_{w} & \mathbf{0} \\
\mathbf{0} & \frac{\rho h^{3}}{12} \mathbf{N}_{\boldsymbol{\Theta}}^{T} \mathbf{N}_{\Theta}
\end{array}\right] d \Omega^{e},
\end{aligned}
$$

where $\Omega^{e}$ is the domain of element $e$, and the two vectors $\boldsymbol{\beta}_{1}=$ $(1,0)^{T}$ and $\beta_{2}=(0,1)^{T}$.
The matrices in Eq. 4 are found by summation of the element matrices, i.e.

$$
\begin{aligned}
\mathbf{K}_{i} & =\sum_{e} \mathbf{k}_{i}{ }^{e}, \\
\mathbf{M} & =\sum_{e} \mathbf{m}^{e} .
\end{aligned}
$$

\section{Appendix B. Group velocity sensitivities}

The derivation is explained in detail below for the $x$-component of the group velocity. Since we specify the frequency, $\omega$, and the angle, $\alpha$, these are considered constant with respect to the density, $\varphi^{e}$, such that

$$
c_{g x}=F_{0}\left(\varphi^{e}, k\left(\varphi^{e}\right), \mathbf{u}\left(\varphi^{e}\right), \mathbf{v}\left(\varphi^{e}\right)\right) .
$$

To make the derivations simpler, we introduce the following normalized eigenvectors

$$
\begin{aligned}
& \mathbf{h}_{1}=\frac{\mathbf{u}}{\sqrt{\mathbf{v}^{T} \mathbf{M u}}}, \\
& \mathbf{h}_{2}=\frac{\mathbf{v}}{\sqrt{\mathbf{v}^{T} \mathbf{M u}}},
\end{aligned}
$$

implying

$F_{0}\left(\varphi^{e}, \omega\left(\varphi^{e}\right), \mathbf{h}_{1}\left(\varphi^{e}\right), \mathbf{h}_{2}\left(\varphi^{e}\right)\right)=\frac{1}{2 \omega} \mathbf{h}_{2}^{T}\left(-i \mathbf{K}_{1}+2 k_{x} \mathbf{K}_{3}+k_{y} \mathbf{K}_{4}\right) \mathbf{h}_{1}$

The derivative with respect to $\varphi^{e}$ is:

$$
\begin{aligned}
\frac{\mathrm{d} F_{0}}{\mathrm{~d} \varphi^{e}} & =\frac{\partial F_{0}}{\partial \varphi^{e}}+\frac{\partial F_{0}}{\partial k} \frac{\mathrm{d} k}{\mathrm{~d} \varphi^{e}}+\frac{\partial F_{0}}{\partial \mathbf{h}_{1}} \frac{\mathrm{d} \mathbf{h}_{1}}{\mathrm{~d} \varphi^{e}}+\frac{\partial F_{0}^{T}}{\partial \mathbf{h}_{2}} \frac{\mathrm{d} \mathbf{h}_{2}}{\mathrm{~d} \varphi^{e}} \\
& =\frac{1}{2 \omega}\left(\mathbf{h}_{2}^{T} \frac{\partial\left(-i \mathbf{K}_{1}+2 k_{x} \mathbf{K}_{3}+k_{y} \mathbf{K}_{4}\right)}{\partial \varphi^{e}} \mathbf{h}_{1}\right. \\
& +\mathbf{h}_{2}^{T} \frac{\partial\left(-i \mathbf{K}_{1}+2 k_{x} \mathbf{K}_{3}+k_{y} \mathbf{K}_{4}\right)}{\partial k} \mathbf{h}_{1} \frac{\mathrm{d} k}{\mathrm{~d} \varphi^{e}} \\
& +\mathbf{h}_{2}^{T}\left(-i \mathbf{K}_{1}+2 k_{x} \mathbf{K}_{3}+k_{y} \mathbf{K}_{4}\right) \frac{\mathrm{d} \mathbf{h}_{1}}{\mathrm{~d} \varphi^{e}} \\
& \left.+\mathbf{h}_{1}^{T}\left(-i \mathbf{K}_{1}+2 k_{x} \mathbf{K}_{3}+k_{y} \mathbf{K}_{4}\right)^{T} \frac{\mathrm{d} \mathbf{h}_{2}}{\mathrm{~d} \varphi^{e}}\right) .
\end{aligned}
$$

To get rid of the implicit derivatives, we need to add three adjoint problems to the original eigenvalue problem

$$
\begin{aligned}
& F_{1}=\mathbf{x}_{1}^{T}\left(\mathbf{K}-\omega^{2} \mathbf{M}\right) \mathbf{h}_{1}=0, \\
& F_{2}=\mathbf{x}_{2}^{T}\left(\mathbf{K}-\omega^{2} \mathbf{M}\right)^{T} \mathbf{h}_{2}=0, \\
& F_{3}=x\left(\mathbf{v}^{T} \mathbf{K u}-\omega^{2} \mathbf{v}^{T} \mathbf{M u}\right)=0,
\end{aligned}
$$

where $\mathbf{x}_{1}, \mathbf{x}_{2}$, and $x$ are arbitrary. That is, they are considered constant in the differentiation. Dividing both sides of $F_{3}$ with $\mathbf{v}^{T}$ Mu yields

$$
F_{3}=x\left(\mathbf{h}_{2}^{T} \mathbf{K} \mathbf{h}_{1}-\omega^{2}\right)=0 .
$$


Adding the adjoint problems to $F_{0}$, differentiating, and gathering terms result in:

$$
\begin{aligned}
\frac{\mathrm{d} c_{g x}}{\mathrm{~d} \varphi^{e}}= & \frac{\mathrm{d}\left(F_{0}+F_{1}+F_{2}+F_{3}\right)}{\mathrm{d} \varphi^{e}}=\frac{\partial F_{0}}{\partial \varphi^{e}}+\frac{\partial F_{1}}{\partial \varphi^{e}}+\frac{\partial F_{2}}{\partial \varphi^{e}}+\frac{\partial F_{3}}{\partial \varphi^{e}}+ \\
& \left(\frac{\partial F_{0}}{\partial k}+\frac{\partial F_{1}}{\partial k}+\frac{\partial F_{2}}{\partial k}+\frac{\partial F_{3}}{\partial k}\right) \frac{\mathrm{d} k}{\mathrm{~d} \varphi^{e}}+ \\
& \left(\frac{\partial F_{0}}{\partial \mathbf{h}_{1}}+\frac{\partial F_{1}}{\partial \mathbf{h}_{1}}+\frac{\partial F_{3}}{\partial \mathbf{h}_{1}}\right) \frac{\mathrm{d} \mathbf{h}_{1}}{\mathrm{~d} \varphi^{e}}+ \\
& \left(\frac{\partial F_{0}^{T}}{\partial \mathbf{h}_{2}}+\frac{\partial F_{2}}{\partial \mathbf{h}_{2}}+\frac{\partial F_{3}^{T}}{\partial \mathbf{h}_{2}}\right) \frac{\mathrm{d} \mathbf{h}_{2}}{\mathrm{~d} \varphi^{e}} .
\end{aligned}
$$

Now, the adjoint variables are chosen such that the terms with implicit derivatives become zero. Resulting in the sensitivities

$$
\begin{aligned}
\frac{\mathrm{d} c_{g x}}{\mathrm{~d} \varphi^{e}} & =\frac{\partial F_{0}}{\partial \varphi^{e}}+\frac{\partial F_{1}}{\partial \varphi^{e}}+\frac{\partial F_{2}}{\partial \varphi^{e}}+\frac{\partial F_{3}}{\partial \varphi^{e}} \\
& =\frac{1}{2 \omega} \mathbf{h}_{2}^{T} \frac{\partial\left(\left(-i \mathbf{K}_{1}+2 k_{x} \mathbf{K}_{3}+k_{y} \mathbf{K}_{4}\right)\right)}{\partial \varphi^{e}} \mathbf{h}_{1}+\mathbf{x}_{1}^{T} \frac{\partial\left(\mathbf{K}-\omega^{2} \mathbf{M}\right)}{\partial \varphi^{e}} \mathbf{h}_{1} \\
& +\mathbf{x}_{2}^{T} \frac{\partial\left(\mathbf{K}-\omega^{2} \mathbf{M}\right)^{T}}{\partial \varphi^{e}} \mathbf{h}_{2}+x \mathbf{h}_{2}^{T} \frac{\partial \mathbf{K}}{\partial \varphi^{e}} \mathbf{h}_{1},
\end{aligned}
$$

when the three adjoint equations are set equal to zero. First adjoint equation:

$$
\begin{aligned}
& \left(\frac{\partial F_{0}}{\partial k}+\frac{\partial F_{1}}{\partial k}+\frac{\partial F_{2}}{\partial k}+\frac{\partial F_{3}}{\partial k}\right)= \\
& \frac{1}{2 \omega} \mathbf{h}_{2}^{T}\left(2 \cos (\theta) \mathbf{K}_{3}+\sin (\theta) \mathbf{K}_{4}\right) \mathbf{h}_{1}+\mathbf{x}_{1}^{T}\left(\frac{\partial \mathbf{K}}{\partial k}\right) \mathbf{h}_{1} \\
& +\mathbf{x}_{2}^{T}\left(\frac{\partial \mathbf{K}}{\partial k}\right)^{T} \mathbf{h}_{2}+x \mathbf{h}_{2}^{T} \frac{\partial \mathbf{K}}{\partial k} \mathbf{h}_{1}=0 .
\end{aligned}
$$

Second adjoint equation:

$$
\begin{aligned}
& \left(\frac{\partial F_{0}}{\partial \mathbf{h}_{1}}+\frac{\partial F_{1}}{\partial \mathbf{h}_{1}}+\frac{\partial F_{3}}{\partial \mathbf{h}_{1}}\right)= \\
& \frac{1}{2 \omega} \mathbf{h}_{2}^{T}\left(-i \mathbf{K}_{1}+2 k_{x} \mathbf{K}_{3}+k_{y} \mathbf{K}_{4}\right)+\mathbf{x}_{1}^{T}\left(\mathbf{K}-\omega^{2} \mathbf{M}\right)+x \mathbf{h}_{2}^{T} \mathbf{K}=\mathbf{0} .
\end{aligned}
$$

Third adjoint equation:

$$
\begin{aligned}
& \left(\frac{\partial F_{0}^{T}}{\partial \mathbf{h}_{2}}+\frac{\partial F_{2}}{\partial \mathbf{h}_{2}}+\frac{\partial F_{3}^{T}}{\partial \mathbf{h}_{2}}\right)= \\
& \frac{1}{2 \omega} \mathbf{h}_{1}^{T}\left(-i \mathbf{K}_{1}^{T}+2 k_{x} \mathbf{K}_{3}^{T}+k_{y} \mathbf{K}_{4}^{T}\right)+\mathbf{x}_{2}^{T}\left(\mathbf{K}-\omega^{2} \mathbf{M}\right)^{T}+x \mathbf{h}_{1}^{T} \mathbf{K}^{T}=\mathbf{0} .
\end{aligned}
$$

Or in matrix form

$$
\begin{gathered}
{\left[\begin{array}{ccc}
\left(\frac{\partial \mathbf{K}}{\partial k}\right) \mathbf{h}_{1} & \left(\mathbf{K}-\omega^{2} \mathbf{M}\right) & \mathbf{0} \\
\left(\frac{\partial \mathbf{K}}{\partial k}\right)^{T} \mathbf{h}_{2} & \mathbf{0} & \left(\mathbf{K}-\omega^{2} \mathbf{M}\right)^{T} \\
\mathbf{h}_{2}^{T} \frac{\partial \mathbf{K}}{\partial k} \mathbf{h}_{1} & \mathbf{h}_{2}^{T} \mathbf{K} & \mathbf{h}_{1}^{T} \mathbf{K}^{T}
\end{array}\right]^{T}\left[\begin{array}{c}
\mathbf{x}_{1} \\
\mathbf{x}_{2} \\
x
\end{array}\right]=} \\
-\left[\begin{array}{cc}
\frac{1}{2 \omega} \mathbf{h}_{2}^{T} & \left(2 \cos (\alpha) \mathbf{K}_{3}+\sin (\alpha) \mathbf{K}_{4}\right) \mathbf{h}_{1} \\
\frac{1}{2 \omega} \mathbf{h}_{2}^{T} & \left(-i \mathbf{K}_{1}+2 k_{x} \mathbf{K}_{3}+k_{y} \mathbf{K}_{4}\right) \\
\frac{1}{2 \omega} \mathbf{h}_{1}^{T} & \left(-i \mathbf{K}_{1}+2 k_{x} \mathbf{K}_{3}+k_{y} \mathbf{K}_{4}\right)^{T}
\end{array}\right]
\end{gathered}
$$

\section{References}

[1] M. M. Sigalas, E. N. Economou, Elastic and acoustic wave band structure, Journal of Sound and Vibration 158 (1992) 377-382.

[2] M. Ruzzene, F. Scarpa, F. Soranna, Wave beaming effects in twodimensional cellular structures, Smart Materials and Structures 12 (2003) 363.

[3] M. Farhat, S. Guenneau, S. Enoch, A. B. Movchan, G. G. Petursson, Focussing bending waves via negative refraction in perforated thin plates, Applied Physics Letters 96 (2010) 081909.

[4] M. Farhat, S. Guenneau, S. Enoch, High directivity and confinement of flexural waves through ultra-refraction in thin perforated plates, EPL (Europhysics Letters) 91 (2010) 54003.

[5] J. Pierre, O. Boyko, L. Belliard, J. O. Vasseur, B. Bonello, Negative refraction of zero order flexural lamb waves through a two-dimensional phononic crystal, Applied Physics Letters 97 (2010) 121919.

[6] L. Hau, S. Harris, Z. Dutton, C. Behroozi, Light speed reduction to 17 metres per second in an ultracold atomic gas, Nature 397 (1999) 594-598.

[7] S. Cox, D. Dobson, Maximizing band gaps in two-dimensional photonic crystals, Siam J Appl Math 59 (1999) 2108-2120.

[8] R. Stainko, O. Sigmund, Tailoring dispersion properties of photonic crystal waveguides by topology optimization, Waves in Random and Complex Media 17 (2007) 477-489.

[9] M. Bendsøe, O. Sigmund, Topology Optimization. Theory, Methods and Applications, Springer, 2003.

[10] L. Brillouin, Wave propagation in periodic structures, 2 ed., Dover Publications, 1953

[11] M. Carrara, M. R. Cacan, M. J. Leamy, M. Ruzzene, A. Erturk, Dramatic enhancement of structure-borne wave energy harvesting using an elliptical acoustic mirror, Applied Physics Letters 100 (2012) 204105.

[12] E. Andreassen, K. Manktelow, M. Ruzzene, Directional bending wave propagation in periodically perforated plates, Journal of Sound and Vibration 335 (2015) 187-203.

[13] O. Sigmund, J. S. Jensen, Systematic design of phononic band-gap materials and structures by topology optimization, Philos. Trans. R. Soc. Lond. A, Math. Phys. Eng. Sci. (UK) Philosophical transactions - Royal Society. Mathematical, Physical and engineering sciences 361 (2003) 1001-1019.

[14] S. Halkjær, O. Sigmund, J. S. Jensen, Inverse design of phononic crystals by topology optimization, Zeitschrift für Kristallographie - Crystalline Materials 220 (2005) 895-905.

[15] S. Halkjær, O. Sigmund, J. S. Jensen, Maximizing band gaps in plate structures, Structural and Multidisciplinary Optimization 32 (2006) 263275.

[16] A. El-Sabbagh, W. Akl, A. Baz, Topology optimization of periodic mindlin plates, Finite Elements in Analysis and Design 44 (2008) 439449.

[17] A. A. Larsen, J. S. Jensen, O. Sigmund, Topological material layout in plates for vibration suppression and wave propagation control, Structural and Multidisciplinary Optimization 37 (2009) 585-594.

[18] R. Haftka, Z. Gürdal, M. Kamat, Elements of Structural Optimization, Kluwer Academic Publishers, 1990.

[19] E. Andreassen, J. S. Jensen, Topology optimization of periodic microstructures for enhanced dynamic properties of viscoelastic composite 
materials, Structural and Multidisciplinary Optimization 49 (2014) 695705.

[20] E. Andreassen, C. S. Andreasen, How to determine composite material properties using numerical homogenization, Computational Materials Science 83 (2014) 488-495.

[21] C. S. Andreasen, E. Andreassen, J. S. Jensen, O. Sigmund, On the realization of the bulk modulus bounds for two-phase viscoelastic composites, Journal of the Mechanics and Physics of Solids 63 (2014) 228-241.

[22] B. Bourdin, B. Bourdin, Filters in topology optimization, International Journal for Numerical Methods in Engineering 50 (2001) 2143-2158.

[23] J. Guest, J. Prevost, T. Belytschko, Achieving minimum length scale in topology optimization using nodal design variables and projection functions, International Journal for Numerical Methods in Engineering 61 (2004) 238-254.

[24] S. Xu, Y. Cai, G. Cheng, Volume preserving nonlinear density filter based on heaviside functions, Structural and Multidisciplinary Optimization 41 (2010) 495-505.

[25] K. Svanberg, Method of moving asymptotes - a new method for structural optimization., International Journal for Numerical Methods in Engineering 24 (1987) 359-373.

[26] F. Wang, B. S. Lazarov, O. Sigmund, On projection methods, convergence and robust formulations in topology optimization, Structural and Multidisciplinary Optimization 43 (2011) 767-784.

[27] O. C. Zienkiewicz, R. L. Taylor, The Finite Element Method. Fifth Edition. Volume 2: Solid Mechanics, Butterworth-Heinemann, 2000. 